\title{
Neutrophil gelatinase-associated lipocalin mediates 13-cis retinoic acid-induced apoptosis of human sebaceous gland cells
}

\author{
Amanda M. Nelson,1,2 Wei Zhao,3 Kathryn L. Gilliland,1,2 \\ Andrea L. Zaenglein, ${ }^{2}$ Wenlei Liu, ${ }^{3}$ and Diane M. Thiboutot ${ }^{1,2}$ \\ ${ }^{1}$ Jake Gittlen Cancer Research Foundation, ${ }^{2}$ Department of Dermatology, and \\ ${ }^{3}$ Department of Health Evaluation Sciences, Pennsylvania State University College of Medicine, Hershey, Pennsylvania, USA.
}

\begin{abstract}
13-cis retinoic acid (13-cis $\mathrm{RA}$; also known as isotretinoin) is the most potent agent available for treatment of acne. It is known that the drug induces apoptosis in cells cultured from human sebaceous glands, but its mechanism of action has not been determined. In this study, skin biopsies were taken from 7 patients with acne prior to and at 1 week of treatment with 13-cis RA. TUNEL staining confirmed that 13-cis RA induced apoptosis in sebaceous glands. Transcriptional profiling of patient skin and cultured human sebaceous gland cells (SEB-1 sebocytes) indicated that lipocalin 2 was among the genes most highly upregulated by 13-cis RA. Lipocalin 2 encodes neutrophil gelatinase-associated lipocalin (NGAL), which functions in innate immune defense and induces apoptosis of murine B lymphocytes. Increased immunolocalization of NGAL was noted in patients' sebaceous glands following treatment with 13-cis RA, and recombinant NGAL induced apoptosis in SEB-1 sebocytes. Furthermore, apoptosis in response to 13-cis RA was inhibited in the presence of siRNA to lipocalin 2. These data indicate that NGAL mediates the apoptotic effect of 13-cis RA and suggest that agents that selectively induce NGAL expression in sebaceous glands might represent therapeutic alternatives to the use of 13-cis RA to treat individuals with acne.
\end{abstract}

\section{Introduction}

13-cis retinoic acid (13-cis $\mathrm{RA}$, also known as isotretinoin) is the most effective drug for the treatment of acne. It is also used to treat pediatric patients with neuroblastoma (1). 13-cis RA is the only retinoid that dramatically reduces the size and secretion of sebaceous glands; yet, its mechanism of action remains largely unknown, in part due to an unexpected anti-acne effect finding during clinical trials in patients with ichthyosis and also the lack of a suitable animal model for acne (2-5). Use of 13-cis RA is limited by its teratogenicity. Because there are no safe alternatives to 13-cis RA that demonstrate comparable efficacy, insights into its mechanism of action are essential for alternative drug discovery.

The goal of the present study is to gain insight into the potential pathways by which 13-cis RA exerts its clearing effect in acne. This is what we believe to be the first study to examine the effects of oral 13-cis RA on gene expression in the skin of acne patients. Array analysis was performed on skin samples that were taken from the backs of acne patients at baseline and after 1 week of 13-cis RA therapy.

One of the most highly upregulated genes was lipocalin 2 (LCN2) that encodes the neutrophil gelatinase-associated lipocalin (NGAL), which has been reported to induce apoptosis $(6,7)$. The actions of LCN2/NGAL were examined in detail in human skin and cultured human sebaceous gland cells (SEB-1 sebocytes). In this paper, we provide what we believe to be the first in vivo evidence that 13-cis RA induces apoptosis within human sebaceous glands and demonstrate that NGAL mediates the apoptotic effect of 13-cis RA on human

Nonstandard abbreviations used: ATRA, all-trans retinoic acid; 13-cis RA, 13-cis retinoic acid; NGAL, neutrophil gelatinase-associated lipocalin; 24p3R, 24p3 receptor; QPCR, quantitative real-time PCR; rhNGAL, recombinant human NGAL.

Conflict of interest: The authors have declared that no conflict of interest exists. Citation for this article: J. Clin. Invest. 118:1468-1478 (2008). doi:10.1172/JCI33869. sebocytes. These data provide important clues that could advance our understanding of retinoid action not only in acne but in other retinoid-responsive conditions such as psoriasis, rosacea, promyelocytic leukemia, and neuroblastoma.

\section{Results}

Patient selection. In an effort to understand the early changes in skin histology and gene expression in response to 13-cis RA, patients were recruited to have skin biopsies of their upper backs at baseline and 1 week into treatment. A total of 7 patients that were prescribed isotretinoin by their dermatologist for their severe acne were enrolled in the study after signing informed consent forms. Information regarding patient age, sex, the time of biopsy, and the dose of isotretinoin that patients were receiving at the time of their second biopsy are presented in Table 1.

Histology suggests decrease in sebaceous gland volume after 1 week of 13-cis $R A$ treatment. H\&E staining was performed on sections of patient skin obtained from biopsies of skin from the upper back taken at baseline and at 1 week of treatment. A total of 17 sections at baseline and 19 sections at 1 week taken from 6 patients were analyzed using image analysis software. At baseline, sebaceous glands were characteristically large and multilobular. Although changes in architecture were not obvious at 1 week of isotretinoin treatment, an early decrease in the overall size of the sebaceous glands was suggested. The mean area of sebaceous glands in the baseline samples was $0.23 \pm 0.09 \mathrm{~mm}^{2}$ (mean \pm SEM) compared with $0.12 \pm 0.02 \mathrm{~mm}^{2}$ in the 1 week samples, which was not significant using a paired $t$ test $(P=0.16)$ (data not shown). Of note is that previous reports noted marked reductions of sebaceous gland size at 16 weeks of treatment (2).

13-cis RA induces apoptosis in patient's sebaceous glands at 1 week. Our previous work indicated that 13-cis RA induced apoptosis within cultured SEB-1 sebocytes (8). To confirm these findings in vivo, a 
Table 1

Isotretinoin patient demographics

\begin{tabular}{lcccc} 
Subject no. & Age & Sex & $\begin{array}{c}\text { Dose } \\
(\mathbf{m g} / \mathbf{k g} / \mathbf{d})\end{array}$ & $\begin{array}{c}\text { Biopsy } \\
\text { (d) }\end{array}$ \\
1 & 15 & $\mathrm{M}$ & 0.5 & 7 \\
2 & 17 & $\mathrm{M}$ & 0.5 & 7 \\
3 & 17 & $\mathrm{M}$ & 0.5 & 7 \\
4 & 21 & $\mathrm{~F}$ & 0.67 & 7 \\
5 & 17 & $\mathrm{M}$ & 0.67 & 7 \\
6 & 20 & $\mathrm{~F}$ & 0.67 & 7 \\
7 & 23 & $\mathrm{M}$ & 0.5 & 7 \\
Mean \pm SD & $18.5 \pm 2.8$ & & $0.57 \pm 0.09$ & $7 \pm 0$ \\
\hline
\end{tabular}

TUNEL-peroxidase assay was performed on skin biopsies obtained from patients at baseline and at 1 week of 13-cis RA therapy $(n=6$ pairs of samples). Patient 7 was omitted from the analysis because no sebaceous glands were captured in the baseline biopsy sections. A 4-fold increase in the percentage of TUNEL-positive cells was noted within the sebaceous gland at 1 week of therapy compared with baseline $(45.9 \% \pm 11.9 \%$ vs. $13.9 \% \pm 6 \%$ TUNEL-positive cells; $P=0.005$, paired $t$ test; $\alpha=0.05$ ) (Figure 1 ). TUNEL staining was strongest in the nuclei of sebocytes in the basal layer of the sebaceous gland and in early differentiated sebocytes adjacent to the basal layer (Figure 1, arrows). Apoptosis was selective for the sebaceous gland, as no apoptosis was detected within the epidermis.

Gene expression analysis of skin from patients treated with 13-cis $R A$ revealed significant increases in genes encoding calcium-binding proteins, retinoid signaling molecules, solute carriers, and serine proteases. Gene array expression analysis was performed on patient skin biopsies at baseline and 1 week of isotretinoin therapy in order to gain insight into putative pathways mediating the apoptotic effect of 13-cis RA. Array data was not generated for patient 3 due to insufficient quantity of RNA. Using a false discovery rate (FDR) of 0.05 that corresponds to a $5 \%$ chance of false-positive gene changes, 38 genes were significantly upregulated and 5 genes were significantly downregulated by 13 -cis RA when compared with baseline (Table 2; see Supplemental Data for a full listing of significantly changed genes; supplemental material available online with this article; doi:10.1172/JCI33869DS1). Many of the significantly upregulated genes are known to be retinoid-responsive genes, including retinoic acid responder 1 (tazarotene induced gene 1 [TIG1]), cellular retinol-binding protein 1 , and cellular retinoic acid-binding protein 2 . Additionally, calcium-binding proteins (i.e., $\mathrm{S} 100$ proteins), serine proteases, serine protease inhibitors (serpins), LCN2, and solute carriers were significantly affected by 13-cis RA. Interestingly, LCN2 was among the most highly upregulated genes. It is an IL-17 target gene that has been reported to induce apoptosis in murine lymphocytes $(6,9)$.

Gene expression in SEB-1 sebocytes treated with 13-cis RA is similar to profile the observed in human skin. Gene expression array analysis performed on patient skin biopsies contains a mixed population of cells. To identify sebocyte-specific gene changes induced by 13-cis RA, we performed array analysis in SEB-1 sebocytes on 3 samples treated with $0.1 \mu \mathrm{M} 13$-cis RA and 3 samples treated with vehicle. Laser-capture microdissection of sebaceous glands from the biopsy specimens was not practical due to the determination that over 200 sections were required per sample to perform the array analysis without using double amplification protocols. A total of 85 genes (78 different genes) were significantly influenced by 13-cis RA: 58 were upregulated and
27 were downregulated. Select genes whose expression was significantly changed are listed in Table 3. (See Supplemental Data for the full listing of significantly changed genes.) As in the patient samples, LCN2 and the tumor suppressor, TIG1, demonstrated the greatest changes in gene expression. In addition, there were changes in several genes involved in apoptosis and innate immunity such as TNF- $\alpha-$ induced protein 2, TRAIL, interferon regulatory factor 1 (IRF1), interferon-induced proteins, $N F \kappa B$, Fas death receptor, and TIG3 (also known as retinoic acid inducible gene 1 [RIG1]). TIG3 encodes an RNA helicase and represents a key intracellular protein that, like TLR3, can recognize viral double-stranded RNA (dsRNA) $(10,11)$.

Quantitative real-time PCR verification of select genes from array analyses. Sufficient RNA was available from 5 of 7 patients (all but Patients 3 and 4) to verify gene expression changes by quantitative realtime PCR (QPCR) for $L C N 2$, retinoic acid receptor responder 1 (RARRES1, TIG1), psoriasin (S100A7), serine protease inhibitor A3 (SERPINA3), and phospholipase A2 group 7 (platelet activating factor acetyl hydrolase) (Figure 2A). For SEB-1 sebocytes, gene array expression changes were verified by QPCR for LCN2, TIG1, insulin-like growth factor-binding protein 3 (IGFBP3), and GATA transcription factor 3 (GATA3) (Figure 2B). With each of the QPCR analyses, the direction and magnitude of the change in expression for the selected genes were similar to those observed with the gene array analyses.

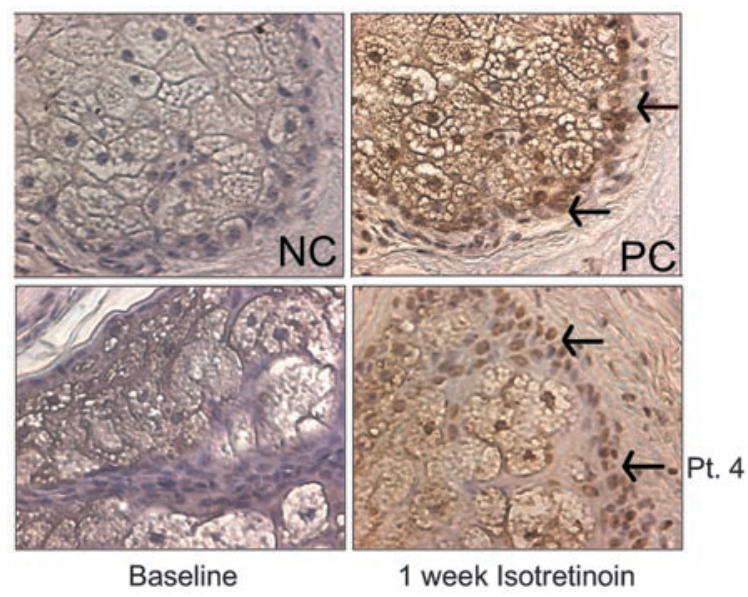

\section{Figure 1}

TUNEL staining is increased in patients' sebaceous glands at 1 week of isotretinoin treatment. Skin sections were obtained from paraffin blocks of 7 patients sampled at baseline and 1 week of isotretinoin and were subjected to TUNEL-peroxidase assay, followed by counterstaining with hematoxylin. At least 2 skin sections from each time point were analyzed from every patient. Patient 7 was omitted from the analysis as no sebaceous glands were found in baseline biopsy sections. Results were quantified by counting TUNEL-positive cells / total cells in sebaceous glands. Representative sections from Patient 4 (Pt. 4) taken at baseline and at 1 week of isotretinoin treatment are shown. TUNEL staining was strongest in the nuclei of sebocytes in the basal layer of the sebaceous gland (arrows) and in early differentiated sebocytes adjacent to the basal layer. Apoptosis was selective for the sebaceous gland, as no apoptosis was detected within the epidermis (not shown). Negative control (NC) consists of sections from a skin specimen treated with DNase I and processed without terminal transferase enzyme. Positive control (PC) consists of sections from a skin specimen treated with DNase I and subjected to the full TUNEL assay. Original magnification, $\times 400$. 


\section{Table 2}

Significantly changed genes in patient skin after 1 week of isotretinoin therapy

\begin{tabular}{lcc} 
Fold change & Gene title & Gene symbol \\
7.03 & lipocalin 2 (oncogene 24p3) & LCN2 \\
6.2 & S100 calcium-binding protein A7 (psoriasin 1) & S100A7 \\
4.53 & S100 calcium-binding protein A9 (calgranulin B) & S100A9 \\
3.78 & solute carrier family 12 (K/Cl transporters) & SLC12A8 \\
3.32 & cytochrome P450, family 2, subfamily B & CYP2B7P1 \\
2.61 & serine (or cysteine) proteinase inhibitor & SERPINA3 \\
2.61 & retinoic acid receptor responder (TIG1) & RARRES1 \\
2.35 & transmembrane protease, serine 4 & TMPRSS4 \\
1.99 & S100 calcium-binding protein P & S100P \\
1.92 & ATPase, H+/K+ transporting, nongastric, alpha & ATP12A \\
1.91 & chemokine (C-C motif) ligand 2 & CCL2 \\
1.81 & retinol-binding protein 1, cellular & RBP1 \\
1.69 & Solute carrier family 6 (amino acid transporter) & SLC6A14 \\
1.67 & E74-like factor 3 (ets domain transcription factor) & ELF3 \\
1.56 & cellular retinoic acid-binding protein 2 & CRABP2 \\
1.52 & defensin, beta 1 & DEFB1 \\
1.51 & calbindin 2, 29kDa (calretinin) & CALB2 \\
1.5 & S100 calcium-binding protein A2 & S100A2 \\
1.49 & involucrin & IVL \\
1.49 & interleukin 27 receptor, alpha & IL27RA \\
-2.29 & Solute carrier family 26, member 3 & SLC26A3 \\
-2.27 & phospholipase A2, group VII (PAF acetylhydrolase) & PLA2G7 \\
-2.13 & phosphodiesterase 6A, cGMP-specific, rod, alpha & PDE6A \\
\hline
\end{tabular}

concentrations ranging from $0.1 \mathrm{nM}$ to $1 \mu \mathrm{M} 13$-cis $\mathrm{RA}$, NGAL protein expression increased in a dose-dependent manner to a maximum of approximately 10 -fold with micromolar concentrations in SEB-1 sebocytes when compared with control $(P<0.05)$ (Figure 4B).

The first 1,000 base pairs of the LCN2 promoter were scanned for retinoic acid response elements (RAREs), using the predefined consensus sequences within the Transfac database through the Transcription Element Search System (TESS), and revealed the presence of both RAR- and RXR-binding sites. Prior studies suggest that 13-cis RA acts as a reservoir of all-trans retinoic acid (ATRA) and, that after isomerization to ATRA, acts via binding nuclear retinoic acid receptors (12). Although, in other studies, retinoid receptor-independent modulation of signaling pathways by ATRA, such as binding to $\mathrm{PKC}$, have also been reported $(13,14)$.

To address this issue, SEB- 1 sebocytes were treated with increasing concentrations of ATRA in parallel studies and examined for expression of LCN2 mRNA and NGAL protein. QPCR and immunoblotting show that NGAL expression also increased in a dose-dependent manner with ATRA when compared with control. The level of NGAL expression induced by ATRA is less than that with 13-cis RA (7-fold vs. 10-fold), but the difference between ATRA and 13-cis RA was not significant (Figure 4).

13-cis RA induces expression of NGAL in patients' sebaceous glands and in SEB-1 sebocytes. The gene LCN2 encodes the protein known as NGAL. Since LCN2 has been reported to be involved in apoptosis and it was among the most highly upregulated genes in both patient skin and in SEB-1 sebocytes, its protein product, NGAL, was chosen for further study as a putative mediator of the apoptotic action of 13-cis RA. Patient skin biopsies that were taken at baseline and at 1 week of isotretinoin therapy were used to assess NGAL expression and localization using immunohistochemistry (Figure 3). In general, NGAL expression is increased after 1 week of isotretinoin treatment, although the amount of increase varied among the patients (Table 4). Patient 7 was omitted from the analysis because no sebaceous glands were captured in the baseline biopsy sections. Interestingly, in 2 out of the 6 patients (Patients 4 and 6, the only females in the study) a minimal increase in NGAL staining was noted. Of the 6 patients examined, only 1 had NGAL expression in sebaceous glands at baseline (Table 4). In each patient, NGAL expression was detected specifically within the basal layer of the sebaceous gland and upper sebaceous duct in sections from skin taken at 1 week of therapy (Figure 3). NGAL expression was not noted within the epidermis. These results are most intriguing when comparing the pattern of NGAL expression with the pattern of TUNEL-positive cells after isotretinoin treatment. NGAL immunoreactivity and TUNEL-positive cells are both present within the basal layer of the sebaceous gland and are absent from the epidermis.

The expression of NGAL within SEB-1 sebocytes was verified by QPCR and western blotting. Expression of LCN2 mRNA was detected after 48 hours of 13-cis RA treatment. 13-cis RA concentrations of $1 \mathrm{nM}$ and greater significantly increased $L C N 2 \mathrm{mRNA}$ levels to a maximum of approximately 6 -fold (with micromolar concentrations) when compared with control (Figure 4A). After 72 hours of treatment with
Recombinant NGAL protein induces apoptosis in SEB-1 sebocytes. Additional studies aimed at testing the hypothesis that NGAL mediates apoptosis in sebaceous glands were performed using SEB-1 sebocytes. SEB-1 sebocytes were treated with increasing concentrations of purified recombinant human NGAL (rhNGAL) protein, and a TUNEL assay was performed. After 24 hours of treatment with NGAL, the percentage of TUNEL-positive cells in SEB-1 sebocytes was significantly increased to a maximum of approximately $35 \%$ using $1 \mathrm{ng} / \mathrm{ml} \mathrm{rhNGAL} \mathrm{(Figure} \mathrm{5).} \mathrm{Interestingly,} \mathrm{this} \mathrm{level} \mathrm{of} \mathrm{apop-}$ tosis is similar to that achieved with 72 hours of 13-cis RA treatment in previous studies (8).

NGAL mediates 13-cis RA-induced apoptosis in human sebocytes. To test the hypothesis that NGAL mediates the apoptotic effect of 13-cis RA on sebocytes, we used siRNA to LCN2 in the presence of 13-cis RA and examined the effect on apoptosis using western blotting for cleaved caspase 3 , the activated form of caspase 3 .

Initially, using QPCR we were able to demonstrate that the expression of LCN2 mRNA was successfully decreased 15-fold (93\%) by the siRNA compared with siCONTROL in SEB-1 cells that were treated for 48 hours with 13-cis RA (Figure 6A). As a control, the specificity of siRNA knockdown was verified using siRNA to GAPDH in parallel samples. GAPDH and LCN2 gene expression was successfully inhibited in their respective samples. In addition, protein levels of NGAL were undetectable by western blotting after 48 and 72 hours of 13-cis RA treatment (Figure 6B).

After confirming successful inhibition of LCN2 and NGAL expression by siRNA, the effect of LCN2 on apoptosis was determined. siRNA to LCN2 in the presence of 13-cis RA decreased expression of cleaved caspase 3 to approximately $20 \%$ of control levels (corresponding to a 5 -fold reduction) (Figure 7). These data indicate that NGAL mediates the apoptotic effect of 13-cis RA in sebocytes. 
Table 3

Selected, significantly changed genes in SEB-1 sebocytes after 13-cis RA treatment

\begin{tabular}{lcc} 
Fold change & Gene title & Gene symbol \\
12.25 & retinoic acid receptor responder (tazarotene induced) 1 & RARRES1 \\
7.04 & lipocalin 2 (oncogene 24p3) & LCN2 \\
5.95 & tumor necrosis factor, alpha-induced protein 2 & TNFAIP2 \\
5.91 & hydroxyprostaglandin dehydrogenase 15-(NAD) & HPGD \\
4.64 & cytochrome P450, family 1, subfamily B, polypeptide 1 & CYP1B1 \\
4.18 & tumor necrosis factor (ligand) superfamily, member 10 & TNFSF10 \\
3.70 & serine (or cysteine) proteinase inhibitor & SERPINB3 \\
3.65 & homeo box A5 & HOXA5 \\
3.43 & insulin-like growth factor-binding protein 3 & IGFBP3 \\
3.29 & aldehyde dehydrogenase 1 family, member A3 & ALDH1A3 \\
3.22 & retinoic acid receptor responder (tazarotene induced) 3 & RARRES3 \\
3.08 & oxidised LDL (lectin-like) receptor 1 & OLR1 \\
2.60 & cyclin-dependent kinase inhibitor 1A (p21, Cip1) & CDKN1A \\
2.51 & E74-like factor 3 (ets domain transcription factor, epithelial-specific ) & ELF3 \\
2.42 & interferon regulatory factor 1 & IRF1 \\
2.42 & interferon-induced protein with tetratricopeptide repeats 3 & IFIT3 \\
2.09 & proteasome (prosome, macropain) subunit, beta type, 10 & PSMB10 \\
2.08 & vascular cell adhesion molecule 1 & VCAM1 \\
2.07 & annexin A9 & ANXA9 \\
2.07 & 2',5'-oligoadenylate synthetase 1, 40/46kDa & OAS1 \\
1.85 & GATA-binding protein 3 & GATA3 \\
1.79 & protein kinase C, alpha & PRKCA \\
1.70 & Fuclear factor of kappa light polypeptide gene enhancer in B-cells & NFKB2 \\
1.69 & Fas (TNF receptor superfamily, member 6) & FAS \\
-4.68 & keratin A & KRT6A \\
-3.94 & FK506-binding protein 5 & FKBP5 \\
-3.25 & ELOVL family member 5, elongation of long chain fatty acids & ELOVL5 \\
\hline &
\end{tabular}

However, isotretinoin, like thalidomide, is a teratogen, and its use is closely regulated through an FDA-mandated risk management program called iPLEDGE. Although isotretinoin has been prescribed for over 20 years, extensive studies into its molecular mechanism of action in human skin and sebaceous glands have not been done. Advances in this regard have been hampered by the lack of an animal model for inflammatory acne. However, studies have shown that within sebaceous glands and sebocyte cell culture (primary cells and immortalized cell lines), 13-cis RA reduces sebaceous lipid production (2, 3, 8, 18-22). 13-cis RA induces apoptosis in SEB-1 sebocytes in addition to inhibiting cell-cycle progression (8).

Using a combination of model systems and methodologies in the present study, we validated that 13-cis RA selectively induces apoptosis in human sebaceous glands, and that this effect is mediated by increased expression of the IL-17 target gene, LCN2. LCN2 encodes the protein, NGAL, which belongs to a family of small molecular weight proteins involved in innate immune defense, retinal transport, prostaglandin synthesis, renal tube morphogenesis, and cellular growth, metabolism, and apoptosis (7, 9, 23-25). Clinically, urine levels of NGAL are used as a marker for acute renal injury (26).

SEB-1 sebocytes express the receptor for NGAL. Recently, a cell surface receptor for the murine homolog to NGAL, 24p3, was identified in murine pro-B lymphocytic FL5.12 cells. The presence of this receptor $(24 \mathrm{p} 3 \mathrm{R})$ is believed to be responsible for cell-specific susceptibility to apoptosis (6). It is possible that NGAL also functions through a cell surface receptor in humans. Based on sequence homology, the human homolog of $24 \mathrm{p} 3 \mathrm{R}$ is predicted to be the solute carrier member, SLC22A17 (6). Expression of SLC22A17 mRNA was detected in SEB-1 sebocytes by QPCR analysis (data not shown). Furthermore, $24 \mathrm{p} 3 \mathrm{R}$ was detected in SEB-1 sebocytes by immunohistochemistry and western blotting, using an antibody to the mouse $24 \mathrm{p} 3 \mathrm{R}$ (Figure 8) (6). Additional studies are required to assess the receptor's involvement in mediating the actions of NGAL.

\section{Discussion}

Acne is the most prevalent skin condition encountered by dermatologists, affecting nearly $85 \%$ of the people between the ages of 12 and 24 years (15). While acne is not life threatening, it does have significant physical and psychosocial morbidity (16). Acne results from the interplay of 4 factors: increased production of sebum by the sebaceous gland, altered keratinization of follicular keratinocytes, activity of Propionibacterium acnes (P. acnes), and inflammation. P. acnes contributes to the inflammation associated with acne via activation of TLR2 on the surface of inflammatory cells in the skin infiltrate (17).

Isotretinoin (13-cis RA) is the most potent agent that affects all the pathogenic features of acne. It is the only therapeutic agent that drastically reduces the size and secretion of sebaceous glands.
NGAL was initially isolated from neutrophils, in which it is complexed with MMP-9 $(27,28)$. In general, NGAL is expressed in tissues exposed to microorganisms such as salivary glands, lung, intestine, uterus, and prostate, in which it may function in innate immune defense against bacterial pathogens $(29,30)$. NGAL is believed to kill bacteria by sequestering iron that is bound to a bacterial siderophore, thus, depriving bacteria of iron that is essential for its survival (7). NGAL expression has been reported within the infundibulum of the hair follicle and follicular epithelium in normal human skin $(31,32)$. We failed, however, to detect NGAL expression in follicles from acne patients prior to isotretinoin therapy. Since NGAL is a secreted protein and acne patients have increased amounts of sebum flowing through the follicle to the skin surface, it is possible that NGAL is secreted onto the surface of the skin and, thus, not detectable using immunohistochemistry.

In the biopsies of skin from patients treated with isotretinoin for 1 week, NGAL localized within the basal layer of the sebaceous gland. Intriguingly, NGAL localization was remarkably similar to the distribution of TUNEL staining, suggesting that NGAL may be responsible for mediating the apoptotic effect of 13-cis RA on sebaceous glands. Increased expression of LCN2 has been linked with the induction of apoptosis in endometrial carcinoma, F12.5 murine lymphocytes, and L929 fibroblasts (6, $33,34)$. Using immunohistochemistry, NGAL expression was increased by only $1 \%$ in female skin compared with $12 \%-36 \%$ in male patients, suggesting possible gender differences in NGAL protein expression in response to 13-cis RA. Clearly additional studies are needed to address this question. In terms of clinical 

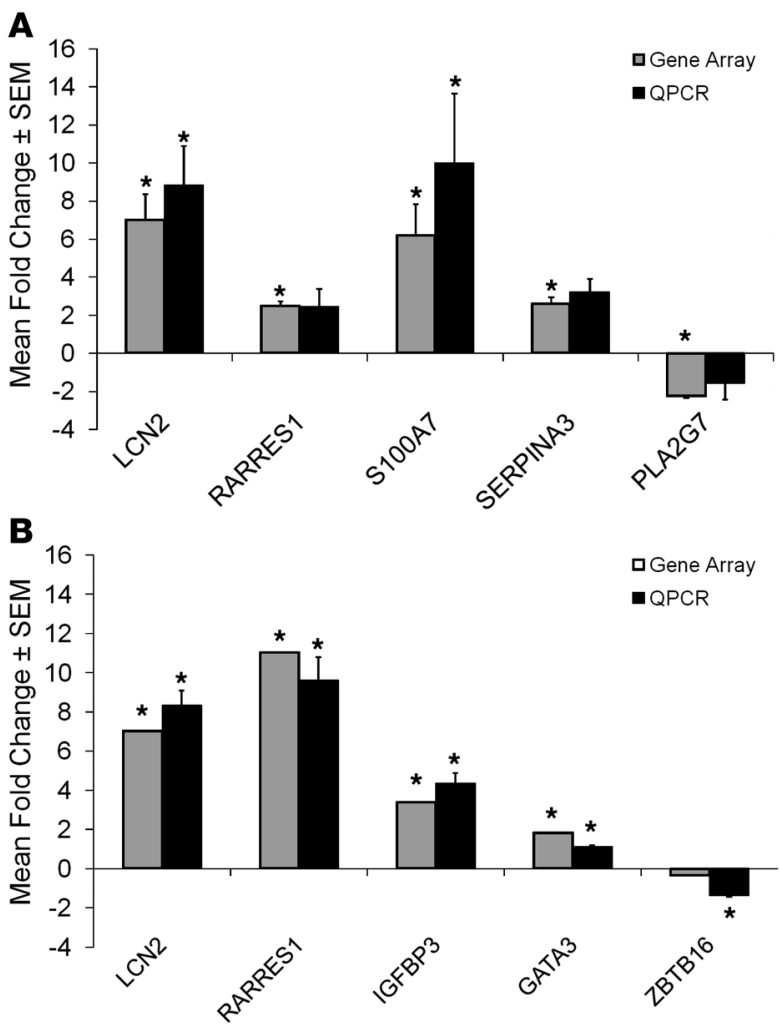

correlation, it is interesting to note that long-lasting remission of acne is less common in adult female patients treated with isotretinoin compared with male patients.

Using SEB-1 sebocytes, we tested the hypothesis that NGAL mediates the apoptotic effect of 13-cis RA. rhNGAL protein induced apoptosis in SEB-1 sebocytes to a similar level that was achieved when these cells were treated with 13-cis RA in previous studies (8). By siRNA, specific knockdown of LCN2 mRNA and NGAL protein expression was achieved in SEB-1 sebocytes treated with 13-cis RA. Apoptosis was decreased by $80 \%$ under these conditions. Taken together, these data indicate that NGAL mediates the apoptotic effect of 13-cis RA on sebaceous glands; however, they do not demonstrate that NGAL is the sole mediator of apoptosis. Gene array analysis indicated that expression of other genes that regulate apoptosis and cell cycle, such as TIG1 and TIG3, was also increased by 13-cis RA $(35,36)$. It is possible that these genes, or others, also play a role in inducing apoptosis in sebocytes.

The mechanism by which NGAL induces apoptosis in sebocytes is unknown. In murine F12 lymphocytes, 24p3 induces apoptosis by binding to a cell surface receptor (24p3R) that functions by shuttling iron out of the cell (6). Database searching of the sequence for the murine receptor led to the identification of the highly conserved human NGAL receptor homolog, SLC22A17 (6). Although, no antibody is available to the human receptor homolog, expression of the 24p3R was confirmed in SEB-1 sebocytes using immunohistochemistry, suggesting that the effects of NGAL in sebocytes could also be receptor mediated.

With involvement of NGAL in diverse cellular processes, including innate immune defense and cell survival, there is

\section{Figure 2}

QPCR verification of gene array changes. In order to verify the direction and magnitude of the changes in gene expression induced by 13-cis RA in the gene microarrays, QPCR was performed using primers to select genes whose expression was significantly changed by 13-cis RA in the array analysis. (A) Comparison of array analysis and QPCR on RNA obtained from patient skin biopsies at baseline and 1 week of 13-cis RA treatment. Data represent the mean \pm SEM of the fold change in gene expression as determined by QPCR in 5 subjects compared to array analysis performed in 6 subjects. RARRES1, retinoic acid receptor responder 1; S100A7, psoriasin; SERPINA3, serine proteinase inhibitor A3; PLA2G7, phospholipase A2 group 7. (B) Comparison of array analysis and QPCR on RNA obtained from SEB-1 sebocytes incubated for 72 hours in the presence or absence of 13-cis RA. Data represent the mean \pm SEM of the fold change in gene expression as determined by QPCR in 8 samples compared to array analysis performed in 3 samples. QPCR results were analyzed by REST-XL software program and ${ }^{*} P<0.05$ was considered significant. IGFBP3, insulin-like growth factor-binding protein 3; GATA3, GATA-binding protein 3; ZBTB16, zinc finger and BTB domain-containing 16.

increasing interest in factors regulating LCN2 expression. Retinoids, such as ATRA and the synthetic retinoid fenretinide, increase the expression of LCN2 in T47D and MCF-7 breast cancer cells (37). 13-cis RA, unlike ATRA and 9-cis RA, is not believed to interact directly with nuclear retinoic acid receptors. It is, however, isomerized to both ATRA and 9-cis RA, which do interact with retinoid receptors (38, 39). Both 13-cis RA and ATRA increased the expression of NGAL in SEB-1 sebocytes in a concentration-dependent manner, 10-fold and 7-fold, respectively. In array studies of normal keratinocytes, 13-cis RA induced an 8-fold increase in LCN2, whereas ATRA and 9-cis RA induced changes on the order of 3-fold and 4-fold, respectively (40).

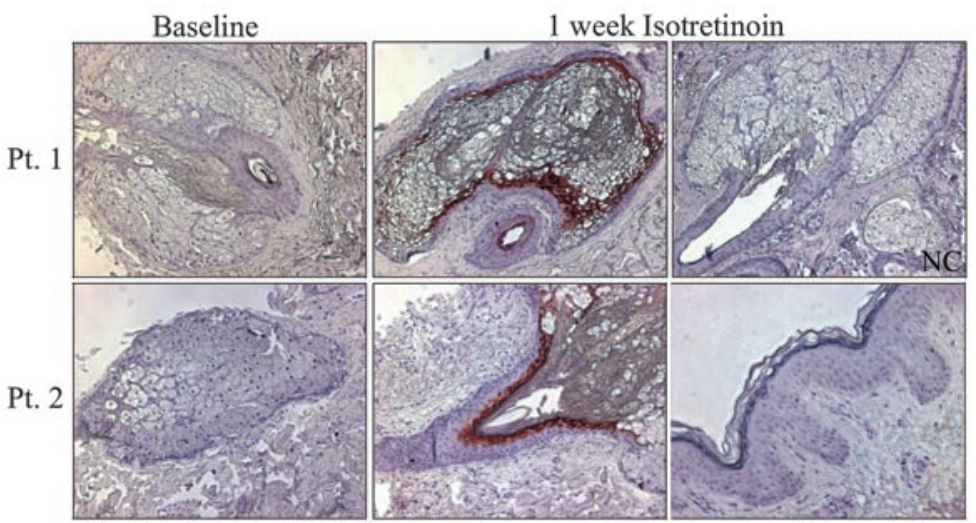

Pt 2. Epidermis

\section{Figure 3}

NGAL expression is increased in sebaceous glands in patients biopsied at 1 week of isotretinoin treatment. Immunohistochemistry using an antibody to NGAL was performed on skin sections taken at baseline and at 1 week of isotretinoin treatment. Sections were incubated overnight with a 1:50 dilution of mouse monoclonal LCN2/NGAL antibody and developed using AEC chromagen (red). All sections were counterstained with hematoxylin. Representative images at baseline and after 1 week isotretinoin from Patients 1 and 2 are shown. An image of the epidermis after isotretinoin treatment from Patient 2 is shown. NGAL was expressed in the sebaceous gland and duct of samples of skin taken at 1 week of isotretinoin therapy. NGAL was not expressed in the epidermis. The amount of NGAL staining varies among patients and individual patient results are shown in Table 4. Negative control consists of normal human skin incubated with normal mouse $\lg _{1}$ antibody. Original magnification, $\times 100$. 


\begin{tabular}{lcc}
$\begin{array}{l}\text { Table } \mathbf{4} \\
\text { Quantification of NGAL staining for individual patients at baseline } \\
\text { and after } 1 \text { week isotretinoin therapy }\end{array}$ \\
\hline Patient & $\begin{array}{c}\text { Baseline (\% of sebaceous } \\
\text { gland positive for NGAL) }\end{array}$ & $\begin{array}{c}\mathbf{1} \text { week (\% of sebaceous } \\
\text { gland positive for NGAL) }\end{array}$ \\
& 0.0 & 36.7 \\
2 & 0.0 & 11.3 \\
3 & 0.0 & 27.1 \\
4 & 0.0 & 1.1 \\
5 & 11.1 & 12.1 \\
6 & 0.0 & 1.0 \\
Average & 1.9 & 14.9 \\
SEM & 1.9 & 5.9
\end{tabular}

Studies are currently underway to determine if the effects of 13-cis RA on LCN2 expression are mediated through its isomers or metabolites or via pathways that are independent of retinoid receptor activation. In addition to retinoids, LCN2 expression is increased by leukotriene B4 and cholesterol oleate, IкB $\zeta$ pathways that are independent of NFKB activation, and IL-17 activation of NFKB dependent pathways $(9,29,41-44)$. Negative regulators of LCN2 expression include iron chelation, activation of the transcription factor JunB, and activation of Wnt signaling pathways $(45,46)$.

LCN2 expression is increased in response to activation of TLR2 and TLR4 signaling by bacterial peptides (42). Although activation of TLR2 by $P$. acnes has been demonstrated in acne lesions, previous studies using gene array failed to indicate increased expression of LCN2 in acne lesions compared with normal skin from the same patients or normal skin from subjects without acne, suggesting that NGAL levels may not be significantly increased by $P$. acnes (17, 47). It is possible that the dramatic increase in NGAL released in response to 13-cis RA targets $P$. acnes as well as inducing apoptosis in the sebaceous gland $(17,48)$. 13-cis RA reduces the numbers of $P$. acnes bacteria on the surface of the skin from acne patients (49, 50). This is thought to result from decreased sebum that acts as a nutrient source for $P$. acnes. However, this reduction in $P$. acnes may be directly related to the induction of NGAL by 13-cis RA. It remains to be determined if NGAL produced in response to 13-cis RA is capable of killing $P$. acnes, if $P$. acnes itself can stimulate NGAL production through TLR2 activation in sebocytes, or if a combination of both processes is occurring.

It was recently demonstrated that LCN2 along with other immunoregulatory genes are transcriptional targets of IL-17 (9). IL-17, an inflammatory cytokine produced by the $\mathrm{CD}^{+}{ }^{+} \mathrm{Th} 17$ subset of $\mathrm{T}$ lymphocytes, is unique in that it provides communication between adaptive immunity and innate immunity to promote inflammation (51). IL-17 has been implicated in the pathogenesis of inflammatory diseases such as collagen-induced arthritis and psoriasis $(52,53)$. Skin from psoriasis patients has increased expression of IL-17 and NGAL within the epidermis in addition to increased levels of endogenous antimicrobial peptides such as active cathelicidin (LL-37) and human $\beta$-defensin 2 (32, 52). IL-17 also triggers expression of human $\beta$-defensin 2 and $M M P 3$, each of which is increased in inflammatory acne lesions compared with unaffected skin $(9,47)$. Activation of NFKB and AP-1 transcription factors increase expression of MMPs (MMP-1, -3, -8, and -9) in acne lesions (54). Although IL-17 regulates gene transcription through $\mathrm{NF \kappa B}$, it remains to be determined whether IL-17 is involved in the induction of LCN2 expression by isotretinoin or in the inflammation associated with acne (9). The promoter region of the LCN2 gene contains consensus sequences for binding retinoic acid receptors (RAR and RXR). It is possible that an isomer or metabolite of 13-cis RA may directly regulate LCN2 expression through interaction with retinoic acid receptors.

Although, they are not the focus of this paper, 13-cis RA increases the expression of several other genes of interest, including S100 proteins (calcium-binding proteins) and serine protease inhibitors (serpins) that have been implicated in innate immune defense (55, 56). S100A7 (psoriasin), which is increased approximately 6-fold by isotretinoin, protects skin against $E$. coli bacterial infections through $\mathrm{Zn}^{+2}$ sequestration and functions as a potent chemoattractant agent for immune cells $(55,57)$. In addition to acne, isotretinoin is used to treat rosacea, an inflammatory skin disease characterized by facial papules and pustules that is often misdiagnosed as acne. Skin from rosacea patients has increased activity of the serine protease kallikrein 5, which generates proinflammatory cathelicidin peptides that have been implicated in the pathogenesis of rosacea (58). Increased expression of serine protease inhibitors by 13-cis RA, which scavenge proinflammatory proteins, may also contribute to the antiinflammatory activity of this drug in rosacea and acne.

The data presented herein suggest that 13-cis RA triggers changes in gene expression that initiate the overall remodeling of the sebaceous gland. The apoptotic effect of 13-cis RA on the sebaceous gland is mediated, at least in part, by the expression of NGAL. These data are the first to our knowledge to indicate an apoptotic effect for NGAL in human cells and suggest that therapeutic strategies that selectively induce NGAL expression in the sebaceous gland may be beneficial in the treatment of acne.

\section{Methods}

Patient selection and tissue biopsies. All protocols were approved by the Institutional Review Board of the Pennsylvania State University College of Medicine and were conducted according to the principles outlined in the Declaration of Helsinki. All subjects gave informed consent. Subjects included males and females, ages $14-40$ years, who were scheduled by their dermatologist to receive treatment with 13-cis RA (isotretinoin, brand not noted) for severe acne. All aspects of the patients' treatment with 13-cis RA apart from the skin biopsies were standard of care and were not part of this research. Exclusion criteria included patients with underlying medical conditions requiring treatment with systemic medications that might interfere with the gene array analysis. Seven patients had 5-mm punch biopsies of skin from the upper back taken at baseline and again at day 7 of treatment. Biopsies were taken from noninflamed skin in areas apart from acne lesions. Biopsies were placed on ice and immediately transferred to the laboratory where they were trimmed of fat, and a small section of each biopsy was taken and paraffin embedded for histology and immunohistochemistry. The remaining portion of the biopsy was flash frozen in liquid nitrogen and used for total RNA isolation.

Image analysis of sebaceous gland size. Following $\mathrm{H} \& \mathrm{E}$ staining of sections from each of the biopsies, image analysis of sebaceous gland size was performed on all available tissue sections. Images were captured using a Spot digital camera (Diagnostic Instruments Inc.), and measurements were obtained with Image Pro Plus Imaging Software version 3.0 after spatial calibration with a micrometer slide under the $\times 10$ objective. All areas of the sebaceous gland were circled using a free-hand measuring tool, and the total area of the sebaceous gland was calculated in each section from the baseline and the 1-week biopsies. The average area for each distinct sebaceous gland was calculated from sections analyzed. A single-factor ANOVA 
A QPCR
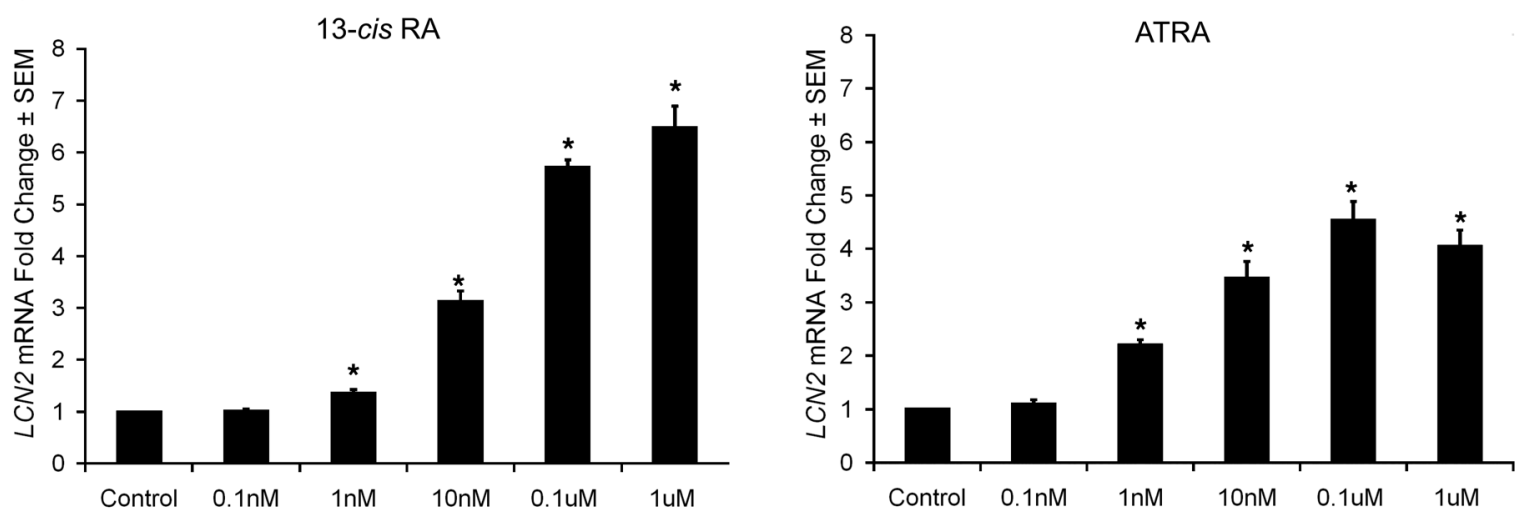

B Western
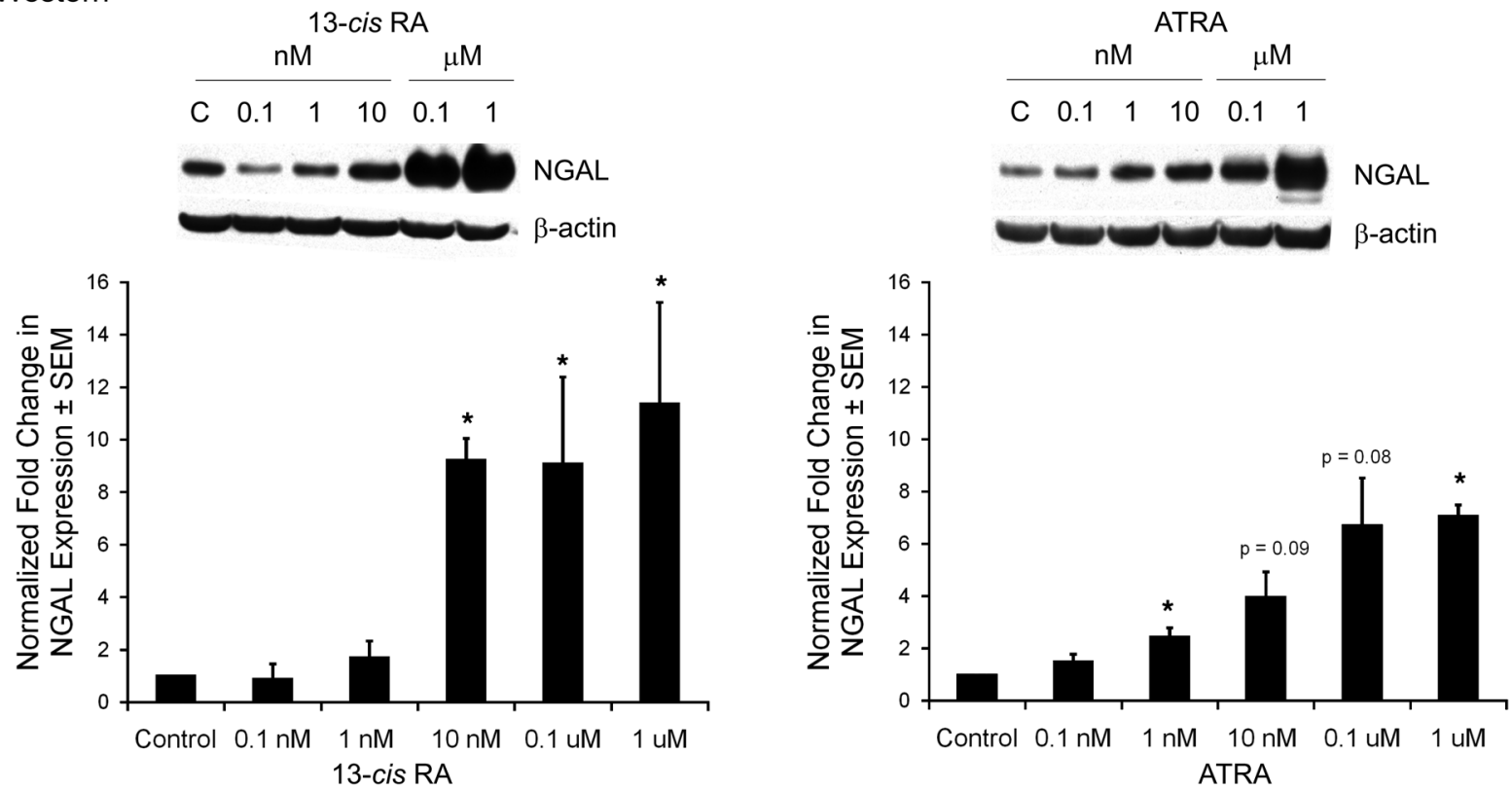

Figure 4

13-cis RA and ATRA increase LCN2 mRNA and NGAL protein expression in SEB-1 sebocytes. SEB-1 sebocytes were treated with vehicle as a control $(C), 13-c i s$ RA $(0.1 \mathrm{nM}, 1 \mathrm{nM}, 10 \mathrm{nM}, 0.1 \mu \mathrm{M}$, or $1 \mu \mathrm{M})$ or ATRA $(0.1 \mathrm{nM}, 1 \mathrm{nM}, 10 \mathrm{nM}, 0.1 \mu \mathrm{M}$, or $1 \mu \mathrm{M})$ for 48 and 72 hours. (A) LCN2 mRNA expression was verified by QPCR after 48 hours of retinoid treatment. Data represent mean \pm SEM of the fold change in gene expression as determined by QPCR of 5 independent samples. Statistical analysis was performed with REST-XL software program and considered significant if ${ }^{\star} P<0.05$. (B) Protein expression was verified by western blot at 72 hours of retinoid treatment. Blots were incubated with primary antibody to NGAL as well as $\beta$-actin as a loading control. Blots were analyzed by densitometry and normalized to $\beta$-actin. The graph represents normalized fold-change values (mean \pm SEM) relative to control for a minimum of 3 independent blots. Statistical analysis was performed with paired $t$ test; ${ }^{*} P<0.05$.

statistical test $(\alpha=0.05)$ was performed to look for significant differences in sebaceous gland area before and after 1 week of treatment.

Cell culture. The SEB-1 human sebocyte cell line was generated by transfection of secondary sebocytes with SV40 large T antigen and was cultured in standard culture medium as previously described (59). 13-cis RA (R 3255) was purchased through Sigma-Aldrich. Stock solutions of 13-cis RA $(10 \mathrm{mM})$ dissolved in ethanol were handled under dimmed yellow light and stored under $\mathrm{N}_{2}$ gas at $-20^{\circ} \mathrm{C}$ until use. Purified rhNGAL protein (amino acids 21-198) was purchased from R\&D Systems ready to use and was stored at $-20^{\circ} \mathrm{C}$ until needed. Stock solutions were diluted to desired concentrations in standard sebocyte culture medium.

Gene expression microarray analysis. Total RNA was isolated from skin biopsies and DNase treated using the RNeasy Fibrous Tissue kit (QIAGEN). Total RNA was isolated from SEB-1 sebocytes treated with $0.1 \mu \mathrm{M}$ 13-cis
RA or vehicle alone $(0.001 \%$ ethanol) in 3 independent samples using the RNeasy kit (QIAGEN). Approximately $2 \mu \mathrm{g}$ of total RNA from each sample was used to generate double-stranded cDNA using a T7-oligo (dT) primer. Biotinylated cRNA, produced through in vitro transcription, was fragmented and hybridized to an Affymetrix human U133A 2.0 microarray for 1-week biopsies and U95Av2 microarray for SEB-1 sebocytes. The arrays were processed on a GeneChip Fluidics Station 450 and scanned on an Affymetrix GeneChip Scanner. Expression signals were normalized as previously described $(47,60,61)$. Significant gene expression alterations were identified using Significance Analysis of Microarrays (SAM) computer software (62). SAM controls the false positives resulting from multiple comparisons through controlling the FDR (63). FDR is defined as the proportion of false-positive genes among all genes that are considered significant. The results of these array analyses have been submitted 
A

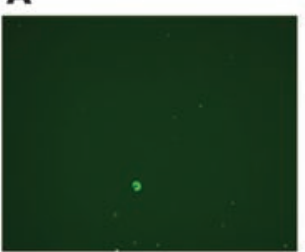

Control

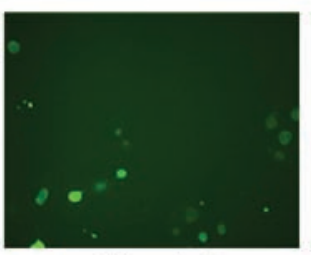

$100 \mathrm{pg} / \mathrm{mL}$

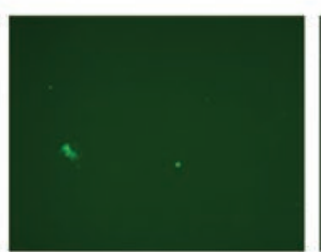

$1 \mathrm{pg} / \mathrm{mL}$

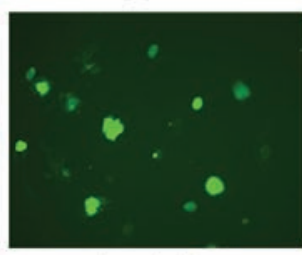

$1 \mathrm{ng} / \mathrm{mL}$

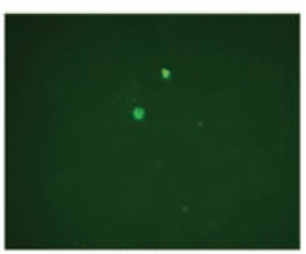

$10 \mathrm{pg} / \mathrm{mL}$

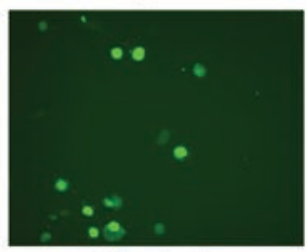

$10 \mathrm{ng} / \mathrm{mL}$
B

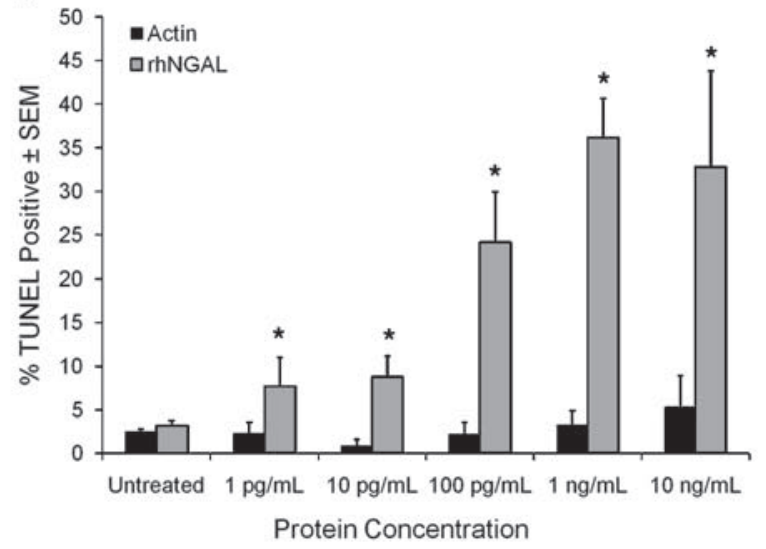

Figure 5

NGAL increases TUNEL staining in SEB-1 sebocytes. SEB-1 sebocytes were treated with vehicle as a control, $1 \mathrm{pg} / \mathrm{ml}, 10 \mathrm{pg} / \mathrm{ml}, 100 \mathrm{pg} / \mathrm{ml}$, $1 \mathrm{ng} / \mathrm{ml}$, or $10 \mathrm{ng} / \mathrm{ml}$ of purified rhNGAL protein (R\&D Systems) or the same concentrations of human actin protein for 24 hours. (A) Representative images of the TUNEL assay using rhNGAL are shown. Original magnification, $\times 200$. (B) Quantification of the percentage of TUNEL-positive stained cells per treatment at 24 hours. Data represent mean \pm SEM; $n=4-8$. Parallel experiments to control for nonspecific effects of protein were performed using human actin protein $(n=2)$. The percentage of TUNEL-positive cells is less than $5 \%$ with all concentrations of actin, which is similar to control values. rhNGAL significantly increased TUNEL staining compared to control over a wide range of concentrations with maximal induction noted at $1 \mathrm{ng} / \mathrm{ml}$. Statistical analyses were performed between vehicle control and each treatment concentration of rhNGAL using 2-factor ANOVA with replication; ${ }^{*} P<0.05$.

to the National Center for Biotechnology Information Gene Expression Omnibus database (GenBank accession no. GSE10434; http://www.ncbi. nlm.nih.gov/geo/).

Quantitative real-time PCR. QPCR experiments confirmed the direction and magnitude of change in selected genes from the microarrays and verified siRNA-knockdown and immunoblotting experiments. Applied Biosystems' Assays-on-Demand Taqman Universal PCR Master Mix, primer/probe sets, and ABI's 7900HT Fast Real-Time PCR System with 384-well plate block module were used according to manufacturer's instructions (Applied Biosystems). Integrity of isolated RNA was verified by agarose gel electrophoresis. cDNA was generated from $1 \mu \mathrm{g}$ of total RNA and primed with oligo-dT, using the SuperScript First-Strand Synthesis System for RT-PCR

\section{Figure 6}

siRNA to $L C N 2$ specifically knocks down $L C N 2$ expression in SEB-1 sebocytes in the presence of 13 -cis RA. SEB-1 sebocytes $\left(2 \times 10^{6}\right.$ cells per $100 \mu$ l reaction mixture) were nucleofected with $1 \mu \mathrm{g}$ siCONTROL, GAPDH (as a specificity control), or LCN2 siRNA. 13-cis RA $(0.1 \mu \mathrm{M})$ was added 24 hours after nucleofection, and cells were incubated for 48 and 72 hours. Extent of siRNA knockdown of gene expression was verified by QPCR and western blotting for LCN2 and GAPDH after 13-cis RA treatment. GAPDH and $L C N 2$ gene expression were successfully inhibited in their respective samples. (A) QPCR analysis of $L C N 2$ and GAPDH mRNA levels at 48 hours of $0.1 \mu \mathrm{M} 13$-cis RA treatment. The expression of LCN2 mRNA was successfully decreased 15-fold by the LCN2 siRNA compared with siCONTROL, whereas expression of GAPDH was minimally affected by siRNA to LCN2. GAPDH mRNA expression was decreased 4-fold by the specific GAPDH siRNA when compared with siCONTROL, whereas siRNA to GAPDH had minimal effects on expression of mRNA for $L C N 2$. Data represent mean $\pm \mathrm{SEM} ; n=6$. ${ }^{*} P<0.05$ as determined by REST-XL program. (B) Western analysis of GAPDH and NGAL protein levels. NGAL protein expression is decreased to undetectable levels by western blotting at 48 and 72 hours of $0.1 \mu \mathrm{M} 13-c i s$ RA treatment with LCN2 siRNA. Representative blots of 4 independent experiments are shown.
(Invitrogen). Diluted cDNA from the 1-week samples were run for the reference gene TATA-binding protein $(T B P)$ and genes of interest $L C N 2$, RARRES1, S100A7, SERPINA3, and PLA2G7. For gene array verification, 8 independent samples of SEB-1 sebocytes treated with $0.1 \mu \mathrm{M}$ 13-cis RA or vehicle alone for 72 hours were analyzed. Genes of interest included LCN2,

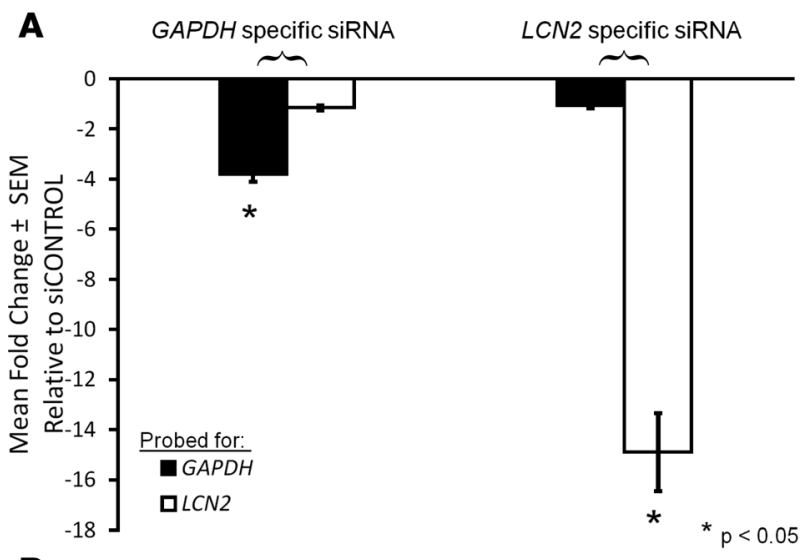

B

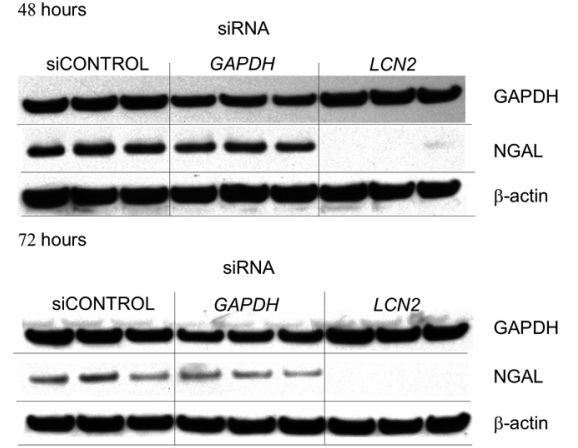



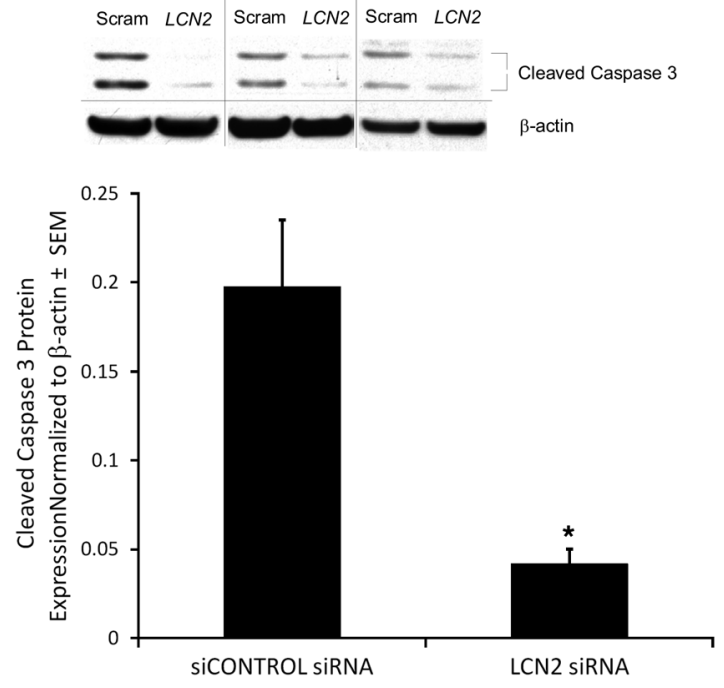

RARRES1, insulin-like growth factor-binding protein 3 (IGFBP3), GATA3, $Z B T B 16$, and potential $L C N 2$ cell surface receptor (SLC22A17). Expression of $T B P, G A P D H$, and $L C N 2$ was analyzed in 6 independent SEB-1 samples for the siRNA-knockdown experiments. SEB-1 sebocytes treated with increasing concentrations of ATRA $(n=5)$ were analyzed for the reference gene $T B P$ and $L C N 2$. In all experiments, assay controls included samples omitting reverse-transcriptase enzyme as well as samples without cDNA. Data was analyzed using the Relative Expression Software Tool (REST-XL version 1) software program (64) with efficiency correction, and a $P$ value less than 0.05 was considered significant.

siRNA. Optimization of the appropriate nucleofection solution and program was carried out according to manufacturer's instructions using the Cell Line Optimization Nucleofector kit (Amaxa Biosystems), with $2 \mu \mathrm{g}$ of pgmaxGFP DNA. The nucleofection solution and program combination that resulted in the highest nucleofection efficiency and lowest cell mortality rate was determined. Program T-20 with solution T was chosen for future experiments. Efficiency of nucleofection was determined by GFP expression quantified by flow cytometry with mock-nucleofected SEB-1 sebocyte controls. Very high levels of GFP expression (87\%, $90 \%, 73 \%$, and $57 \%$ ) were detected at 24, 48, 72 and 96 hours after nucleofection, respectively.

Using ON-TARGET plus Smartpool Human LCN2, Human GAPDH, and siCONTROL siRNA duplex oligonucleotides (Dharmacon Research), nucleofection was performed as suggested by Dharmacon and Amaxa Biosystems. SEB- 1 sebocytes, $2 \times 10^{6}$ cells per $100 \mu \mathrm{l}$ reaction, were nucleofected with $1 \mu \mathrm{g}$ siCONTROL, GAPDH, or LCN2 siRNA. 13-cis RA $(0.1 \mu \mathrm{M})$ was added 24 hours after nucleofection. Extent of siRNA knockdown of gene expression was verified by QPCR and western blotting for $L C N 2 / \mathrm{NGAL}$ and GAPDH at various time points after 13-cis RA treatment. Involvement of LCN2/NGAL in mediating 13-cis RA induced apoptosis was assessed by cleaved caspase 3 protein expression using western blotting.

NGAL and 24p3R immunohistochemistry. Immunohistochemistry was performed on formalin-fixed paraffin-embedded human skin sections using the avidin-biotin complex method and AEC development (ABC kit and AEC Substrate kit for Peroxidase; Vector Laboratories). Baseline and 1-week-treatment patient biopsy sections were subjected to deparaffinization, rehydration, and antigen retrieval with TRILOGY buffer (Cell Marque) prior to immunohistochemistry. Sections were incubated overnight with mouse monoclonal LCN2 antibody (Abcam Inc.). Negative controls were prepared with normal mouse $\mathrm{IgG}_{1}$ antibody (SCBT; Santa Cruz). Sections were counterstained with hematoxylin using standard procedures.

\section{Figure 7}

siRNA to NGAL blocks the induction of cleaved caspase 3 by 13-cis RA in SEB-1 sebocytes. Under siRNA conditions described in Figure 6, immunoblotting revealed that siRNA to $L C N 2$ decreases expression of cleaved caspase 3 by approximately 5 -fold compared with siRNA control. Blots were incubated with primary antibodies to cleaved caspase 3 as well as $\beta$-actin as a loading control to normalize densitometry values. Three independent scrambled siCONTROL (SCRAM) and LCN2 western blots are shown. p17 and p19 are the cleaved or active fragments of caspase 3. Graph represents the normalized expression of cleaved caspase 3 from 5 independent western blots. These data indicate that NGAL mediates the apoptotic response of SEB-1 sebocytes to 13-cis RA. Statistical analysis was performed with paired $t$ test, mean $\pm \mathrm{SEM} ;{ }^{*} P<0.05$.

For quantification of NGAL, Image Pro Plus Imaging Software version 3.0 was used to measure the area of "red" (NGAL positive) compared with the total area of the sebaceous gland in 2 sections for each patient $(n=6)$. Area of NGAL / total area sebaceous gland was calculated in baseline and 1-week-treatment sections for each patient.

$24 \mathrm{p} 3 \mathrm{R}$ (NGAL receptor) affinity purified antibody was kindly provided by Michael Green (University of Massachusetts Medical School, Worcester, Massachusetts, USA) (6). SEB-1 sebocytes were cultured and fixed according to standard procedures followed by overnight incubation with $24 \mathrm{p} 3 \mathrm{R}$ antibody or normal rabbit IgG (negative control) and counterstaining with hematoxylin.

TUNEL staining. Sections of baseline and posttreatment biopsies from 7 patients were subjected to deparaffinization, rehydration, and permeabilization with $0.1 \%$ Triton- $X$ and $0.1 \%$ sodium citrate in PBS. Sections were subjected to In Situ Cell Death Detection, Peroxidase kit (Roche Diagnostics) followed by counterstaining with hematoxylin. DNase I treatment of positive (with terminal transferase) and negative (without

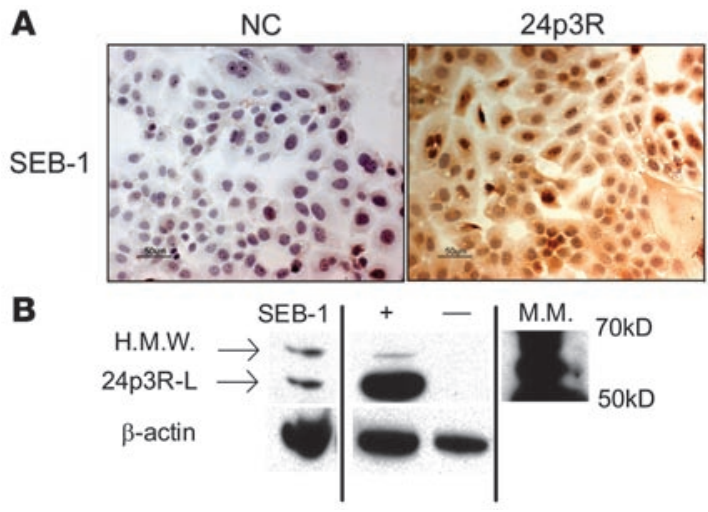

\section{Figure 8}

SEB-1 sebocytes express the receptor for NGAL. (A) SEB-1 sebocytes were grown under standard conditions and immunocytochemistry was performed using an antibody to the murine 24p3R. Slides were counterstained with hematoxylin. Negative control (NC) was processed with normal rabbit IgG antibody in place of the primary antibody. Scale bar: $50 \mu \mathrm{m}$. Immunoreactivity for the 24p3R localizes to the cytoplasm of SEB-1 sebocytes. (B) Western analysis confirms presence of the receptor and indicates 2 receptor isoforms are present in SEB-1 sebocytes: high molecular weight (H.M.W.) and 24p3R long (24p3R-L). Positive ([+]; HEK 293 cell lysate) and negative ([-]; T47D cell lysate) controls provided by are shown. All samples were run on the same gel but were noncontiguous. Blot shown is representative of 3 independent experiments. 
terminal transferase) controls were included as assay controls according to manufacturer's instructions. At least 2 baseline and 1-week skin sections were analyzed from every patient. Patient 7 was omitted from the analysis, as no sebaceous glands were found in baseline biopsy sections. Results were analyzed by counting positively stained cells and dividing by the total cells in sebaceous glands. Data represent mean \pm SD, $n=6$ patients; paired $t$ test was used for statistical analysis and considered significant if $P$ values were less than 0.05 .

SEB-1 sebocytes (passage 22-28) were cultured in 12-well plates in standard medium until approximately $30 \%-40 \%$ confluent. Wells were rinsed with PBS and treated in duplicate with vehicle control, $1 \mathrm{pg} / \mathrm{ml}, 10 \mathrm{pg} / \mathrm{ml}$, $100 \mathrm{pg} / \mathrm{ml}, 1 \mathrm{ng} / \mathrm{ml}$, or $10 \mathrm{ng} / \mathrm{ml}$ of rhNGAL (R\&D Systems) or human actin (negative control; Cytoskeleton) protein for 24 hours. In all experiments, each well was considered 1 sample. Samples were prepared by manufacturer's instructions for In Situ Cell Death Detection Kit, Fluorescein (Roche Diagnostics). DNase I treatment of positive and negative controls (without terminal transferase) were included as assay controls according to manufacturer's instructions. Results were analyzed and quantified by counting positive stained cells per total number of cells in 3 representative fields per well for each of the treatments done in duplicate. Four independent rhNGAL experiments were performed, whereas treatment with actin was performed once. Statistical analyses were performed between control and each treatment concentration, using 2-factor ANOVA with replication and considered significant if $P$ was less than 0.05 .

Western blotting. Mouse monoclonal antibody to LCN2 (NGAL) was obtained from Abcam Inc. The 24p3R (NGAL receptor) affinity-purified antibody, positive (HEK 293 cell lysate) and negative (T47D cell lysate) controls were kindly provided by Michael Green. GAPDH (no. 2118), cleaved caspase 3 (no. 9664), $\beta$-actin (no. 4967) and anti-rabbit HRP linked secondary antibodies (no. 7074) were purchased from Cell Signaling Technology. Secondary antimouse HRP antibody was purchased from Santa Cruz Biotechnology Inc.

SEB-1 sebocytes (passages 22-28) were in standard culture medium until 50\%-75\% confluent. Treatments of 13-cis RA ( $0.1 \mathrm{nM}, 1 \mathrm{nM}, 10 \mathrm{nM}$, $0.1 \mu \mathrm{M}$, or $1 \mu \mathrm{M}$,) or ethanol vehicle $(0.01 \%$ or less) were applied for 48 or 72 hours. In parallel studies, identical concentrations of ATRA were applied for 72 hours. In all experiments, each plate was considered an independent sample, and total protein was collected. Blots were incubated with appropriate primary and secondary antibodies followed by densitometry. Each experiment was repeated a minimum of 3 independent times. Data was analyzed using a paired $t$ test, and results were considered significant if $P$ was less than 0.05 .

\section{Acknowledgments}

The authors wish to thank Trey Bruggeman, Loren Clarke, and Nishit Trivedi for assistance with histology of skin biopsies and Amy Longenecker and Silvia Gosik for coordination of patient procedures. Special thanks to Michael Green and Claude Gazin (University of Massachusetts Medical School, Worcester, Massachusetts, USA) for kindly providing the $24 \mathrm{p} 3 \mathrm{R}$ antibody and control lysates. This study was supported by NIH NIAMS R01 AR47820 to D.M. Thiboutot, NIH General Clinical Research Center Grants M01RR010732 and C06RR016499 to the Pennsylvania State University College of Medicine, the Jake Gittlen Cancer Research Foundation, and the Department of Dermatology at the Pennsylvania State University College of Medicine.

Received for publication September 7, 2007, and accepted in revised form January 16, 2008.

Address correspondence to: Diane M. Thiboutot, Department of Dermatology, HU14, Pennsylvania State University College of Medicine, 500 University Drive, Hershey, Pennsylvania 17033, USA. Phone: (717) 531-7437; Fax: (717) 531-4821; E-mail: dthiboutot@psu.edu.

Wenlei Liu's present address is: Eli Lilly \& Co., Indianapolis, Indiana, USA.

Wei Zhao's present address is: Kaplan Financial, Indianapolis, Indiana, USA.
1. Reynolds, C.P., Matthay, K.K., Villablanca, J.G., and Maurer, B.J. 2003. Retinoid therapy of high-risk neuroblastoma. Cancer Lett. 197:185-192.

2. Landthaler, M., Kummermehr, J., Wagner, A., and Plewig, G. 1980. Inhibitory effects of 13-cis retinoic acid on human sebaceous glands. Arch. Dermatol. Res. 269:297-309.

3. Strauss, J.S., Stranieri, A.M., Farrell, L.N., and Downing, D.T. 1980. The effect of marked inhibition of sebum production with 13-cis retinoic acid on skin surface lipid composition. J. Invest. Dermatol. 74:66-67.

4. Goldstein, J.A., Comite, H., Mescon, H., and Pochi, P.E. 1982. Isotretinoin in the treatment of acne. Arch. Dermatol. 118:555-558.

5. Peck, G.L. 1979. Prolonged remissions of cystic acne with 13-cis retinoic acid. N. Engl. J. Med. 300:329.

6. Devireddy, L.R., Gazin, C., Zhu, X., and Green, M.R. 2005. A cell-surface receptor for lipocalin $24 \mathrm{p} 3$ selectively mediates apoptosis and iron uptake. Cell. 123:1293-1305.

7. Flo, T.H., et al. 2004. Lipocalin 2 mediates an innate immune response to bacterial infection by sequestrating iron. Nature. 432:917-921.

8. Nelson, A.M., Gilliland, K.L., Cong, Z., and Thiboutot, D.M. 2006. 13-cis Retinoic acid induces apoptosis and cell cycle arrest in human SEB-1 sebocytes. J. Invest. Dermatol. 126:2178-2189.

9. Shen, F., Hu, Z., Goswami, J., and Gaffen, S.L. 2006. Identification of common transcriptional regulatory elements in interleukin-17 target genes. J. Biol.
Chem. 281:24138-24148.

10. Yoneyama, M., et al. 2004. The RNA helicase RIG-I has an essential function in double-stranded RNAinduced innate antiviral responses. Nat. Immunol. 5:730-737.

11. Sen, G.C., and Sarkar, S.N. 2005. Hitching RIG to action. Nat. Immunol. 6:1074-1076.

12. Tsukada, M., et al. 2000. 13-cis retinoic acid exerts its specific activity on human sebocytes through selective intracellular isomerization to all-trans retinoic acid and binding to retinoid acid receptors. J. Invest. Dermatol. 115:321-327.

13. Ochoa, W.F., et al. 2003. Retinoic acid binds to the C2-domain of protein kinase C(alpha). Biochemistry. 42:8774-8779.

14. Pettersson, F., Couture, M.-C., Hanna, N., and Miller, W., Jr. 2004. Enhanced retinoid-induced apoptosis of MDA-MB-231 breast cancer cells by PKC inhibitors involved activation of ERK. Oncogene. 23:7053-7066

15. White, G.M. 1998. Recent findings in the epidemiologic evidence, classification, and subtypes of acne vulgaris. J. Am. Acad. Dermatol. 39:S34-S37.

16. Bickers, D.R., et al. 2006. The burden of skin diseases: 2004: A joint project of the American Academy of Dermatology Association and the Society for Investigative Dermatology. J. Am. Acad. Dermatol. 55:490-500.

17. Kim, J., et al. 2002. Activation of toll-like receptor 2 in acne triggers inflammatory cytokine responses. J. Immunol. 169:1535-1541.
18. Zouboulis, C.C., Zia, L., Korge, B., Gollnick, H., and Orfanos, C.E. 1991. Cultivation of human sebocytes in vitro: cell characterization and influence of synthetic retinoids. In Retinoids: 10 years on. J.-H. Saurat, editor. S. Karger Publishers. Basel, Switzerland. 254-273.

19. Zouboulis, C.C., et al. 1991. Effects of 13-cis retinoic acid, all-trans-retinoic acid, and acitretin on the proliferation, lipid synthesis and keratin expression of cultured human sebocytes in vitro. J. Invest. Dermatol. 96:792-797.

20. Zouboulis, C.C., Seltmann, H., Neitzel, H., and Orfanos, C.E. 1999. Establishment and characterization of an immortalized human sebaceous gland cell line (SZ95). J. Invest. Dermatol. 113:1011-1020.

21. Gomez, E.C., and Moskowitz, R.J. 1980. Effect of 13-cis retinoic acid on the hamster flank organ. J. Invest. Dermatol. 74:392-397.

22. Goldstein, J.A., et al. 1982. Comparative effect of isotretinoin and etretinate on acne and sebaceous gland secretion. J. Am. Acad. Dermatol. 6:760-765.

23. Newcomer, M.E., and Ong, D.E. 2000. Plasma retinol binding protein: structure and function of the prototypic lipocalin. Biochim. Biophys. Acta. 1482:57-64.

24. Yang, J., et al. 2002. An iron delivery pathway mediated by a lipocalin. Mol. Cell. 10:1045-1056.

25. Hanai, J., et al. 2005. Lipocalin 2 diminishes invasiveness and metastasis of Ras-transformed cells. J. Biol. Chem. 280:13641-13647.

26. Mishra, J., et al. 2003. Identification of neutrophil 
gelatinase-associated lipocalin as a novel early urinary biomarker for ischemic renal injury. J. Am. Soc. Nephrol. 14:2534-2543.

27. Kjeldsen, L., Bainton, D.F., Sengelov, H., and Borregaard, N. 1994. Identification of neutrophil gelatinase-associated lipocalin as a novel matrix protein of specific granules in human neutrophils. Blood. 83:799-807.

28. Yan, L., Borregaard, N., Kjeldsen, L., and Moses, M.A. 2001. The high molecular weight urinary matrix metalloproteinase (MMP) activity is a complex of gelatinase B/MMP-9 and neutrophil gelatinaseassociated lipocalin (NGAL). Modulation of MMP-9 activity by NGAL. J. Biol. Chem. 276:37258-37265.

29. Akerstrom, B., Flower, D.R., and Salier, J.P. 2000. Lipocalins: unity in diversity. Biochim. Biophys. Acta. 1482:1-8.

30. Logdberg, L., and Wester, L. 2000. Immunocalins: a lipocalin subfamily that modulates immune and inflammatory responses. Biochim. Biophys. Acta. 1482:284-297.

31. Seo, S.J., et al. 2006. Expression of neutrophil gelatinase-associated lipocalin in skin epidermis. J. Invest. Dermatol. 126:510-512.

32. Mallbris, L., et al. 2002. Neutrophil gelatinaseassociated lipocalin is a marker for dysregulated keratinocyte differentiation in human skin. Exp. Dermatol. 11:584-591.

33. Lin, H.H., Li, W.W., Lee, Y.C., and Chu, S.T. 2007. Apoptosis induced by uterine $24 \mathrm{p} 3$ protein in endometrial carcinoma cell line. Toxicology. 234:203-215

34. Li, P.T., Lee, Y.C., Elangovan, N., and Chu, S.T. 2007. Mouse 24p3 protein has an effect on L929 cell viability. Int. J. Biol. Sci. 3:100-107.

35. Nagpal, S., et al. 1996. Tazarotene-induced gene 1 (TIG1), a novel retinoic acid receptor-responsive gene in skin. J. Invest. Dermatol. 106:269-274.

36. DiSepio, D., et al. 1998. Identification and characterization of a retinoid-induced class II tumor suppressor/growth regulatory gene. Proc. Natl. Acad. Sci. U. S. A. 95:14811-14815.

37. Caramuta, S., et al. 2006. Regulation of lipocalin2 gene by the cancer chemopreventive retinoid 4-HPR. Int. J. Cancer. 119:1599-1606.

38. Levin, A., Bosakowski, T., Kazmer, S., and Grippo, J.F. 1992. 13-cis retinoic acid does not bind to retinoic acid receptors alpha, beta and gamma [abstract]. Toxicologist. 12:181.

39. Allenby, G., et al. 1993. Retinoic acid receptors and retinoid $X$ receptors: interactions with endogenous retinoic acids. Proc. Natl. Acad. Sci. U. S. A. 90:30-34.

40. Baron, J.M., et al. 2005. Retinoic acid and its 4-Oxo metabolites are functionally active in human skin cells in vitro. J. Invest. Dermatol. 125:143-153.

41. Kjeldsen, L., Cowland, J.B., and Borregaard, N. 2000. Human neutrophil gelatinase-associated lipocalin and homologous proteins in rat and mouse. Biochim. Biophys. Acta. 1482:272-283.

42. Draper, D.W., Bethea, H.N., and He, Y.W. 2006. Toll-like receptor 2-dependent and -independent activation of macrophages by group B streptococci. Immunol. Lett. 102:202-214.

43. Cowland, J.B., Sorensen, O.E., Sehested, M., and Borregaard, N. 2003. Neutrophil gelatinase-associated lipocalin is up-regulated in human epithelial cells by IL-1 beta, but not by TNF-alpha. J. Immunol. 171:6630-6639.

44. Fujino, R.S., et al. 2006. Spermatogonial cell-mediated activation of an IkappaBzeta-independent nuclear factor-kappaB pathway in Sertoli cells induces transcription of the lipocalin-2 gene. Mol. Endocrinol. 20:904-915.

45. Ziegler, S., et al. 2007. Lipocalin $24 \mathrm{p} 3$ is regulated by the Wnt pathway independent of regulation by iron. Cancer Genet. Cytogenet. 174:16-23.

46. Florin, L., et al. 2004. Identification of novel AP-1 target genes in fibroblasts regulated during cutaneous wound healing. Oncogene. 23:7005-7017.

47. Trivedi, N.R., Gilliland, K.L., Zhao, W., Liu, W., and Thiboutot, D.M. 2006. Gene array expression profiling in acne lesions reveals marked upregulation of genes involved in inflammation and matrix remodeling. J. Invest. Dermatol. 126:1071-1079.

48. Jugeau, S., et al. 2005. Induction of toll-like receptors by Propionibacterium acnes. Br. J. Dermatol. 153:1105-1113.

49. Leyden, J.J., McGinley, K.J., and Foglia, A.N. 1986. Qualitative and quantitative changes in cutaneous bacteria associated with systemic isotretinoin therapy for acne conglobata. J. Invest. Dermatol. 86:390-393.

50. Coates, P., et al. 2005. Efficacy of oral isotretinoin in the control of skin and nasal colonization by antibiotic-resistant propionibacteria in patients with acne. Br. J. Dermatol. 153:1126-1136.

51. Kawaguchi, M., Adachi, M., Oda, N., Kokubu, F., and Huang, S.K. 2004. IL-17 cytokine family. J. Allergy Clin. Immunol. 114:1265-1273.

52. Li, J., Chen, X., Liu, Z., Yue, Q., and Liu, H. 2007. Expression of Th17 cytokines in skin lesions of patients with psoriasis. J. Huazhong Univ. Sci. Technolog. Med. Sci. 27:330-332.

53. Lubberts, E., et al. 2001. IL-1-independent role of IL-17 in synovial inflammation and joint destruction during collagen-induced arthritis. J. Immunol. 167:1004-1013

54. Kang, S., et al. 2005. Inflammation and extracellular matrix degradation mediated by activated transcription factors nuclear factor-kappaB and activator protein-1 in inflammatory acne lesions in vivo. Am. J. Pathol. 166:1691-1699.

55. Eckert, R.L., et al. 2004. S100 proteins in theepidermis. J. Invest. Dermatol. 123:23-33.

56. Silverman, G.A., et al. 2001. The serpins are an expanding superfamily of structurally similar but functionally diverse proteins. Evolution, mechanism of inhibition, novel functions, and a revised nomenclature. J. Biol. Chem. 276:33293-33296.

57. Glaser, R., et al. 2005. Antimicrobial psoriasin (S100A7) protects human skin from Escherichia coli infection. Nat. Immunol. 6:57-64.

58. Yamasaki, K., et al. 2007. Increased serine protease activity and cathelicidin promotes skin inflammation in rosacea. Nat. Med. 13:975-980.

59. Thiboutot, D., et al. 2003. Human skin is a steroidogenic tissue: Steroidogenic enzymes and cofactors are expressed in epidermis, normal sebocytes, and an immortalized sebocyte cell line (SEB-1). J. Invest. Dermatol. 120:905-914.

60. Irizarry, R.A., Gautier, L., and Cope, L.M. 2003. An $\mathrm{R}$ package for analyses of affymetrix oligonucleotide arrays. In The analysis of gene expression data: methods and software. G. Parmigiani, E.S. Garrett, R.A. Irizarry, and S.L. Zeger, editors. Springer Verlag. New York, New York, USA. 102-119.

61. Irizarry, R.A., et al. 2003. Exploration, normalization, and summaries of high density oligonucleotide array probe level data. Biostatistics. 4:249-264.

62. Tusher, V.G., Tibshirani, R., and Chu, G. 2001. Significance analysis of microarrays applied to the ionizing radiation response. Proc. Natl. Acad. Sci. U.S. A. 98:5116-5121.

63. Benjamini, Y., and Yekutieli, D. 2005. Quantitative trait Loci analysis using the false discovery rate. Genetics. 171:783-790.

64. Pfaffl, M.W., Horgan, G.W., and Dempfle, L. 2002. Relative expression software tool (REST) for groupwise comparison and statistical analysis of relative expression results in real-time PCR. Nucleic Acids Res. 30:e36. 